\title{
Clinical and socio-demographic factors associated with electroencephalographic abnormalities in children with epilepsy
}

\author{
Christian Chukwukere Ogoke, ${ }^{1}$ Wilson Chukwuneke Igwe, ${ }^{2}$ Esther Ngozi Umeadi \\ ${ }^{1}$ Consultant Paediatrician \& Child Neurologist, Paediatric Neurology Unit, Department of Paediatrics, King \\ Fahd Central Hospital, Jizan, Saudi Arabia; ${ }^{2}$ Department of Paediatrics, Faculty of Medicine, Nnamdi \\ Azikiwe University, Awka, Nigeria
}

\begin{abstract}
Electroencephalography (EEG) makes critical contribution to the management of epilepsies. Few studies have examined the clinical and socio-demographic factors that are likely to predict finding an abnormal or epileptiform EEG in children with epilepsy (CWE). Knowledge of clinical variables and socio demographic factors that determine EEG abnormalities may inform careful selection of children for EEG and improve the cost-effectiveness
\end{abstract}

\footnotetext{
Correspondence: Wilson Chukwuneke Igwe, Department of Paediatrics, Faculty of Medicine, Nnamdi Azikiwe University, PMB 5025, Awka, Anambra State,Nigeria.

Tel.: +2347036531408

Email: wc.igwe@unizik.edu.ng
}

Key words: clinical and socio-demographic factors, children with epilepsy, epileptiform EEG

Acknowledgements: We acknowledge the staff of Mother Healthcare Diagnostics \& Hospital, Owerri for their assistance at the EEG laboratory both during EEG recording and data collection.

Conflict of interest: The authors have no conflict of interest to declare.

Availability of data and materials: All data generated or analyzed during this study are included in this published article.

Ethics approval and consent to participate: This research was approved by the Federal Medical Centre Owerri Health Research Ethics Committee (approval number: FMC/OW/HREC/232) before commencement of the study. All patients participating in this study signed a written informed consent form for participating in this study.

Informed consent: Written informed consent was obtained from a legally authorized representative(s) for anonymized patient information to be published in this article.

Received for publication: 2 July 2021

Revision received: 14 August 2021.

Accepted for publication: 14 August 2021

This work is licensed under a Creative Commons Attribution NonCommercial 4.0 License (CC BY-NC 4.0).

CC Copyright: the Author(s),2020

Licensee PAGEPress, Italy

Annals of Clinical and Biomedical Research 2021; 2:154

doi:10.4081/acbr.2021.154 of this investigation. Therefore, this study was carried out to assess the relationship between the occurrence of EEG abnormalities and certain factors such as age, gender, clinical neurologic state, seizure type, anti-epileptic drug therapy, activation procedure such as sleep and etiology of epilepsy in children who had EEG in Owerri, Nigeria. The clinical and EEG records of children who had EEG at a tertiary referral center over a period of two years were retrospectively reviewed. Relevant data including demographics, clinical neurologic state, seizure type, EEG findings were extracted. Statistical analysis was used to determine association between categorical variables. The variables noted to be significantly associated with abnormal EEG recordings were the presence of abnormal neurologic findings $(p=0.020)$ and etiology of epilepsy $(p=0.045)$. There were no significant association between abnormal EEG findings and age $(p=0.680)$, gender $(p=0.802)$ and seizure types $(\mathrm{p}=0.157)$. The clinical neurological state and etiology of epilepsy in children were significantly associated with the occurrence of abnormalities and yield of interictal scalp EEG. Children with epilepsy who are neurologically abnormal or have multiple etiological factors could be prioritized in EEG appointments in resource-poor settings. Further research in children with epilepsy is needed to confirm these findings.

\section{Introduction}

Epilepsy is a significant neurological disease in children due to its chronicity, cost of treatment and negative effects on cognitive function and overall development. ${ }^{1}$ The incidence and prevalence of epilepsy in children is highest in rural areas of underdeveloped countries. ${ }^{2}$ Expenditure on drugs and laboratory investigations contribute most to the cost of treatment of epilepsy. ${ }^{3}$ The economic cost of treatment of epilepsy in children is high and often beyond the rich of many households in Nigeria. ${ }^{3}$

Electroencephalography remains the most important investigation for children with epilepsy since it aids diagnosis and management by differentiating seizure types and epilepsy syndromes, aiding appropriate choice of drugs and predicting risk of seizure recurrence. ${ }^{4}$ However, EEG has low sensitivity and frequently needs to be repeated to increase chances of recording epileptiform abnormalities. ${ }^{4}$ In resource-poor settings, EEG and other neurodiagnostic tests are unavailable, inaccessible and unaffordable. ${ }^{5}$ Therefore, careful patient selection may improve the cost-effectiveness of EEG.

Little is known about factors that are associated with abnormal EEG or clinical variables that predict EEG abnormalities in children with epilepsy. The aim of this study was to identify the factors that are associated with abnormal EEG in children with epilepsy. 


\section{Materials and Methods}

Study site

The study was carried out at the Electrophysiology laboratory/Child Neurology Clinic of Mother Healthcare Diagnostics \& Hospital, Owerri. The Mother Healthcare Diagnostics \& Hospital is a multi-specialist hospital and diagnostic centre with an EEG unit/Child Neurology Clinic that was the first to commence paediatric digital EEG recording in Owerri, South east Nigeria. It serves as a referral centre for paediatric EEG for most hospitals in Owerri and the Child Neurology Clinic caters for children with neurological disorders. This was a retrospective review of the clinical and EEG records of all children with epilepsy who were seen at the facility over a two-year period.

\section{Ethical approval}

This research was approved by the Federal Medical Centre Owerri Health Research Ethics Committee (approval number: FMC/OW/HREC/232) before commencement of the study.

\section{Study population}

The study population consisted of children aged $<18$ years who were seen at the facility between January 2017 and December 2018. Children who had febrile seizures, normal EEG after single unprovoked seizures, or those whose clinical and EEG findings supported Non-Epileptic Paroxysmal Events (NEPEs) were excluded from the study. Neonates were not seen during the period under review.

\section{Study protocol}

Each patient's clinical history, physical examination findings, Basic Data Sheet (Appendix) for EEG, EEG findings and other investigation results including neuroimaging of the brain, if done, were reviewed and relevant data carefully recorded on a pro forma. EEG recording was done in an air-conditioned electrophysiology laboratory after application of electrodes with electrolyte paste on the scalp according to the 10-20 International system (where applicable, this was modified). With the electrodes connected to the inputs of the differential amplifier (NCC MEDICAL ${ }^{\circledR}$ Nation $7128 \mathrm{~W} /$ Nation $7128 \mathrm{WH}$, Type C EEG system) and the single channel EKG also connected, digital recording was done for a minimum of thirty minutes. Sleep was frequently induced using oral melatonin both to relax the child and as an additional activation procedure. Intermittent eye opening and closure, hyperventilation, intermittent photic stimulation and other standard protocols such as recording in three different montages (at least one referential and one bipolar) were all observed. The waveforms were visually inspected on the desktop monitor and interpreted by a clinical neurophysiologist with specific training in EEG interpretation in children. Each EEG was classified as normal or abnormal based on the presence or absence of background abnormalities (disorganization, asymmetry/focal slowing, generalized slowing, excessive beta activity, background attenuation/suppression e.t.c) or specific epileptiform abnormalities (spikes, polyspikes, sharp waves and their respective complexes). Interpretation in a clinical context was done by integrating the EEG findings and all relevant information in the Basic Data Sheet (Appendix). Diagnosis and classification of epilepsy types based on the etiology, EEG findings and semiol-

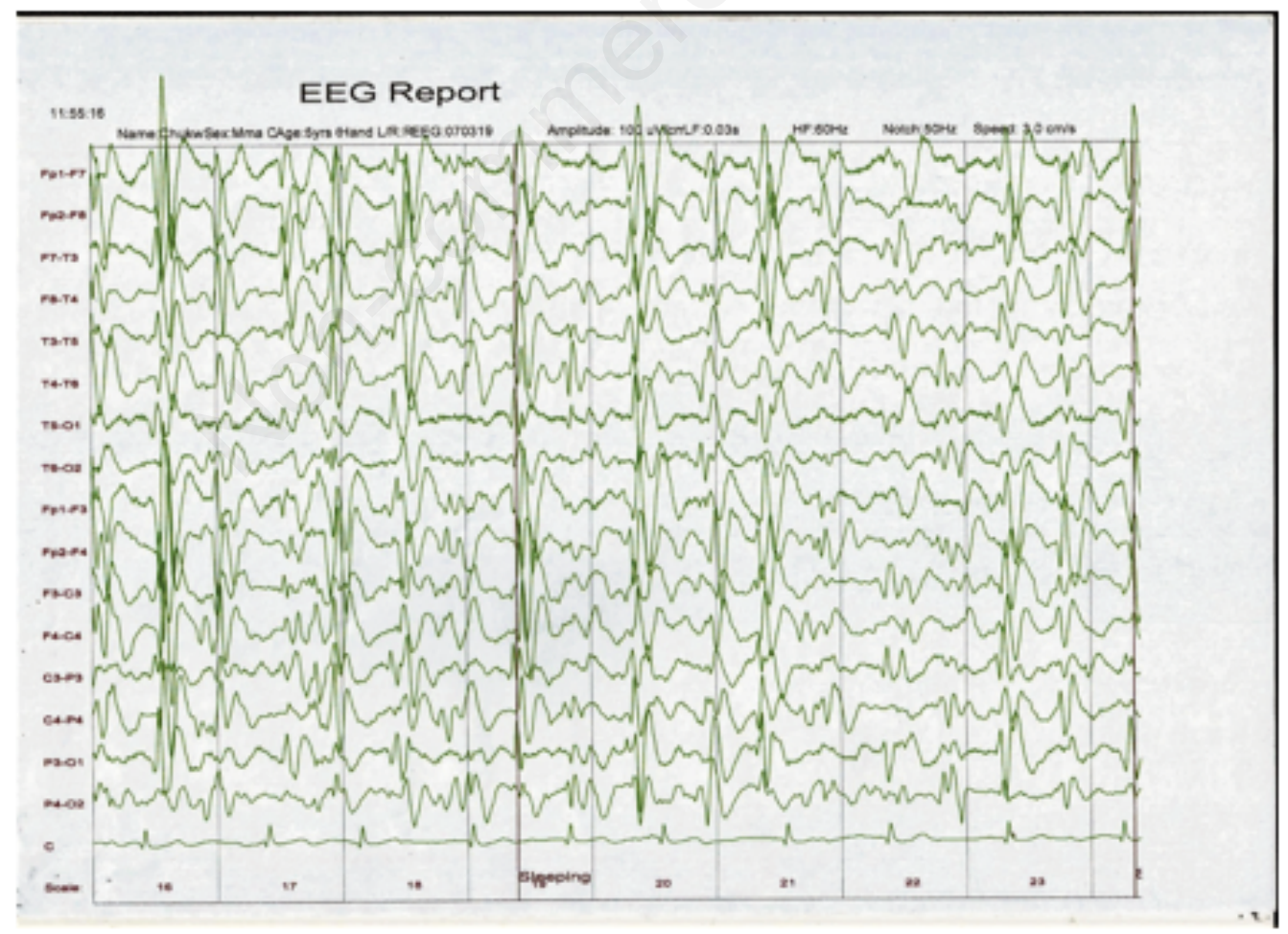

Figure 1. A severely abnormal record showing in sleep continuous generalized high amplitude slow (1 - $2 \mathrm{~Hz})$ sharp- and spike - slow wave complexes (GSWD) in a 5 year-old boy with tonic, atonic and atypical absence seizures, abnormal development and behavior following an acute encephalopathy/complicated meningitis at age of 13 months. CT brain showed in this child showed a non-enhancing wedge shaped parasagittal hypodensity in the right parietal lobe suggestive of Rt. Parietal lobe infarct. A diagnosis of Lennox-Gastaut syndrome was made and aetiology documented as structural. 
ogy, and epilepsy syndrome were done according to the current 2017 International League Against Epilepsy (ILAE) recommendations. ${ }^{6,7}$ In the etiologic classification in this study, a structural etiology was accepted based on abnormal neuroimaging findings of CT scan or MRI. Those who had a probable structural cause for their epilepsy such as head injury with impact seizures, focal seizures and focal background slowing on EEG but had no brain imaging (CT or MRI) were categorized as multiple/unknown. Thus, an appreciable proportion of patients with post traumatic epilepsy fell under the unknown category. Patients categorized as having a genetic etiology had a relevant family history, typical seizure semiology, normal pregnancy, birth history, normal development, normal neurological examination and normal EEG background. Those classified as having an infectious etiology were neurologically normal prior to the onset of the central nervous system infections.

Children with a history suggestive of severe birth asphyxia (prolonged/obstructed labor, not crying immediately after birth, with or without post asphyxia seizures within 24-48 hours post delivery, Apgar scores of 3 and below) without any neuroimaging studies were categorized as unknown since the cortical injury due to asphyxia was not proven. However some of these had microcephaly or speech/language disorder that suggested cortical injury.

See Figure 1 and 2 for examples of abnormal EEG.

\section{Data analysis}

Data were analyzed using the statistical package for the social sciences (SPSS version 25; IBM Corp; USA). The categorical variables such as age, gender, clinical neurological state, clinical seizure type, anti-epileptic drug therapy, additional activation procedure (sleep) and the etiology of epilepsy were examined for association with abnormal EEG using Pearson's chi square test. Statistical significance was set at $\mathrm{p}<0.05$.

\section{Results}

\section{Characteristics of study population}

Out of a total of 123 EEGs done during the two-year period under review, 8 patients had incomplete data; of the remaining 115 , epilepsy was diagnosed in 97 children. This comprised 57 (58.8\%) males and $40(41.2 \%)$ females giving a M:F gender ratio of $1.43: 1$. Their ages ranged $0.33-17.67$ years with a mean $\pm(\mathrm{SD})$ of $5.7 \pm 4.4$ years. A past history of meningitis, head trauma with impact seizures and family history of seizures were obtained in $7(7.2 \%)$, $4(4.2 \%)$ and $65(67 \%)$ of the children respectively. Developmental delay and regression were established in $25(25.8 \%)$ and $5(5.2 \%)$ respectively while $14(14.4 \%)$ and $5(5.2 \%)$ had microcephaly and macrocephaly respectively. Neuroimaging (CT/MRI) was done in only $12(12.4 \%)$ of the patients.

\section{EEG findings}

EEG was normal in 33 (34\%) of the children, abnormal in 52 $(53.6 \%)$ and were not interpretable in $12(12.45)$ because of poor quality. EEG was epileptiform in $36(37.1 \%)$ and non-epileptiform in $49(50.5 \%)$. The EEG abnormalities seen were background abnormalities (disorganization, asymmetry/focal slowing, generalized slowing, excessive beta activity, background attenuation/sup-

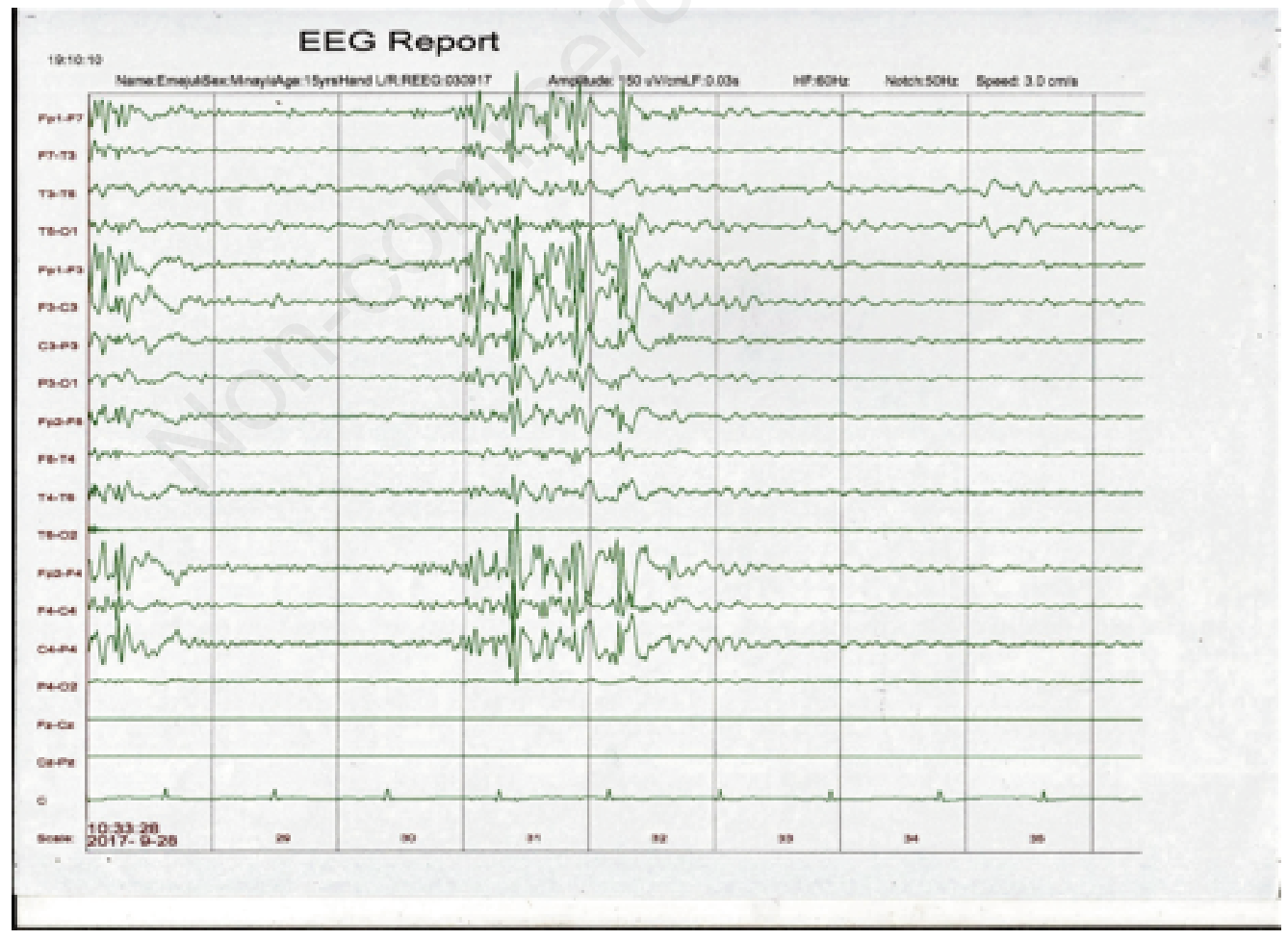

Figure 2. An abnormal inter-ictal EEG (at decreased sensitivity) with normal background activity and paroxysmal, fast, generalized spikes and polyspike-wave complexes (GPSWD) in a 16 year-old male with only infrequent GTCSs occurring on awakening (at 7a.m, 5 a.m) provoked by fasting, attending vigil church services and short lasting sleep at nights. No history of myoclonic jerks or absences. He had a positive family history of epileptic seizures and a first-grade academic performance. A diagnosis of Epilepsy with GTCSs only was made and aetiology was documented as genetic. 
pression) and specific epileptiform abnormalities (spikes, polyspikes, sharp waves and their respective complexes). These EEG findings made epilepsy syndrome diagnosis possible in $38(39 \%)$ of the children. The syndromes were: Benign Rolandic Epilepsy, Panayiotopoulos syndrome, Watanabe-Vigevano syndrome, West syndrome, Childhood Absence Epilepsy, Idiopathic Generalized Epilepsy with generalized tonic-clonic seizures only and Janz syndrome.

\section{Association between abnormal EEG and categorical variables \\ Age and abnormal EEG}

The occurrence of abnormal EEG was analyzed in relation to the age of the patients. The proportion of children with abnormal EEG was highest in those $<5$ years but difference in proportions was found not to be statistically significant $(\mathrm{p}=0.680)$ (Table I).

\section{Gender and abnormal EEG}

The proportion of males with abnormal EEG was similar to that of females and the gender of the children in relation to abnormal EEG was not statistically significant indicating no association between them $(\mathrm{p}=0.802$, Table 1$)$.

\section{Clinical neurological state and abnormal EEG}

A significantly higher proportion $(69.4 \%)$ of children in the cohort had a normal neurological state. However, the proportion of children with neurologic abnormalities who had EEG abnormalities was about 1.5 times more than that of children with no neurological conditions. The association between abnormal EEG and neurologic status was found to be statistically significant $(\mathrm{p}=0.020$, Table 1).

\section{Clinical seizure type and abnormal EEG}

There was no significant association between the occurrence of focal, generalized, or unknown-onset seizures and abnormal EEG $(\mathrm{p}=0.157$, Table 1$)$.

\section{Anti-Epileptic Drug (AED) therapy and abnormal EEG}

A slightly higher proportion of children who were on AED therapy had abnormal EEG compared to those not taking medications. The occurrence of EEG abnormalities and AED therapy showed no statistically significant association $(p=0.450$, Table 1$)$.

\section{Activation procedure (sleep) and abnormal EEG}

Similar proportions of children with drug-induced sleep as an additional activation procedure and those with awake records had abnormal EEG. There was no association between the occurrence of abnormalities on EEG and induction of sleep $(p=0.780$, Table 1).

\section{Aetiology of epilepsy and abnormal EEG}

Children with unknown/multiple aetiological factors were more likely to have abnormal EEG findings. There was a statistically significant association between the occurrence of structural, genetic, infectious and unknown/multiple aetiological factors and abnormal EEG ( $\mathrm{p}=0.045$, Table 1$)$.

\section{Discussion}

This is the first study on factors associated with abnormal EEG in Children With Epilepsy (CWE) in our locality. In general, there is a dearth of studies on the factors associated with abnormal or epileptiform EEG in CWE. Knowledge of such factors would pos-

Table 1. Association between EEG outcome and categorical variables.

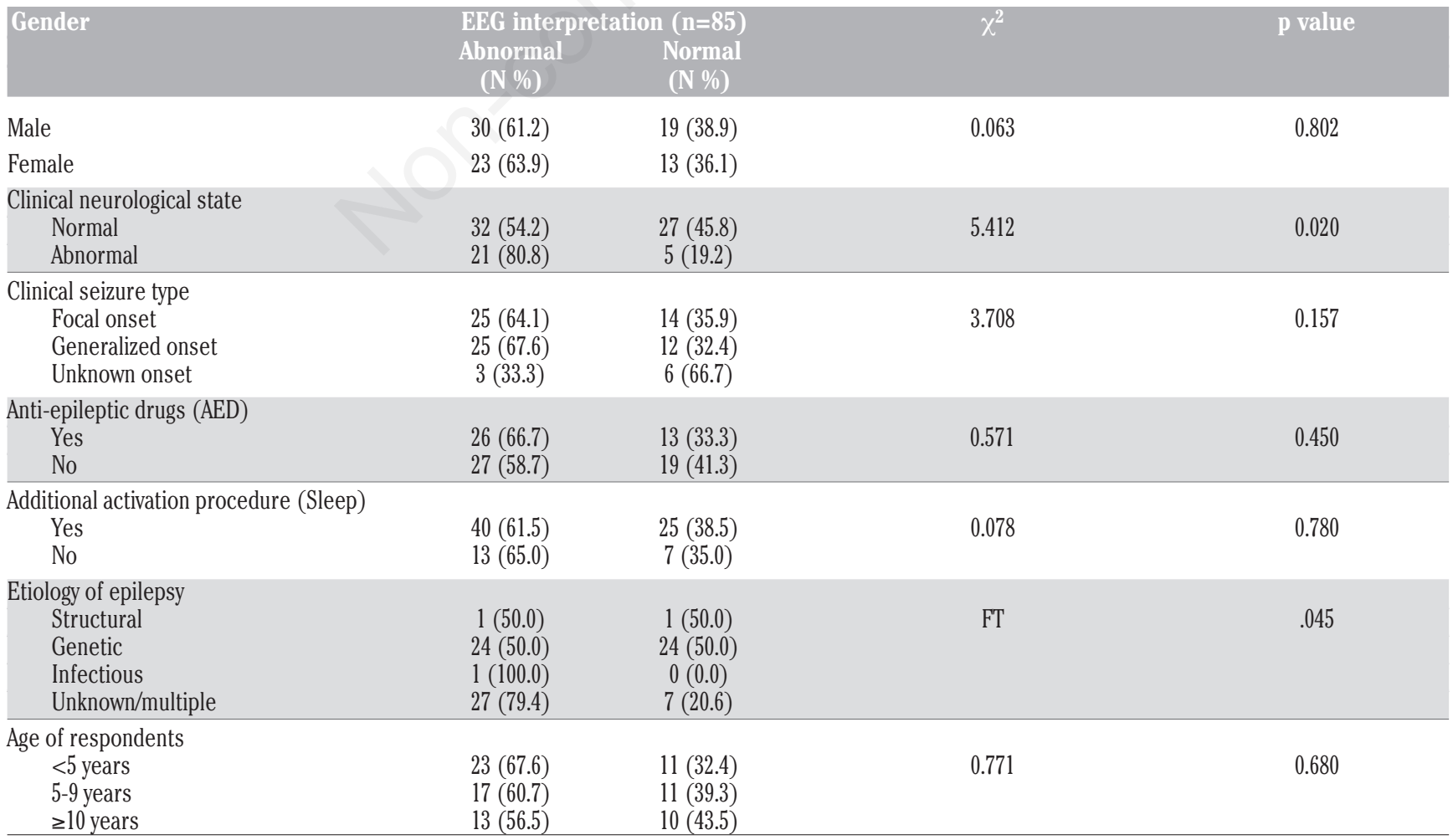

FT: Fisher exact Test. 
sibly facilitate a cost-effective diagnostic work-up of patients with unprovoked afebrile seizures. This study has shown that the occurrence of abnormal or epileptiform EEG in CWE were associated with abnormal neurological condition and etiology of epilepsy but no correlation with age, gender, clinical seizure type, AED therapy and additional activation procedure. This implies that the majority of clinical and socio-demographic characteristics in CWE are not likely to improve patient selection and cost-effectiveness of EEG. Similar studies ${ }^{8-11}$ have given conflicting reports. However those studies were done exclusively in adults and in both adults and children.

A significantly higher proportion of children (69.4\%) had normal clinical neurological state and suggests a high prevalence of genetic epilepsies in the cohort since children with genetic epilepsies such as Benign Rolandic Epilepsy (BRE), Childhood Absence Epilepsy (CAE), Panayiotopoulos syndrome, e.t.c are usually neurologically normal. The observation that an abnormal neurologic state was more likely to be associated with epileptiform EEG in this study is similar to finding by Aina et al. ${ }^{12}$ in South west Nigeria that earlier reported a high prevalence of epileptiform EEG abnormalities in children with neurodevelopmental disorders due to the associated structural and metabolic brain damage resulting from perinatal insults and cerebral malformation. These brain abnormalities underlie EEG abnormalities of background slowing or excessive neuronal discharges (hypersynchronous activity). This is further supported by the finding in this present study of the under 5 age group showing the highest correlation with abnormal EEG since neurodevelopmental disorders occur in early childhood. However, Aina et al. reported no statistically significant relationship between EEG abnormalities and clinical diagnosis (developmental abnormalities). In a large multi-centre study by Kariuki et $a l .{ }^{13}$ there was no association between abnormal EEG and neurological deficits across all the five sites in Africa. Thus the significant association between abnormal EEG and clinical neurological state reported in this present study differs from these reports ${ }^{12,14}$ and is difficult to explain. Other studies ${ }^{8-11}$ did not evaluate this factor (clinical neurological state or neurological deficits) in relation to occurrence of abnormal or epileptiform EEG. Therefore, more studies in children with epilepsy are needed due to the conflicting reports. However, the implication of this finding is that CWE who present with hard neurological signs such as microcephaly and macrocephaly or developmental delay or regression should be prioritized in EEG appointments in resource-poor settings.

Likewise, the relationship between the occurrence of abnormal EEG and etiology of epilepsy has not been evaluated by earlier studies and thus it is difficult to say if the positive association reported in this study is a valid finding. Nevertheless, abnormalities are very likely to be seen on EEG in certain epilepsies like Childhood Absence Epilepsy and other Idiopathic Generalized Epilepsies (currently called genetic epilepsies) and epileptic encephalopathies like West or Lennox Gastaut syndrome. ${ }^{4}$ These epilepsies have different etiological factors and if certain syndromes of certain etiologies are already known to frequently show EEG abnormalities, an association between etiology of epilepsy and the occurrence of EEG abnormalities as found in this study truly exists. In the present study, the children classified under unknown or multiple etiological factors were either not fully investigated because of finances, had multiple risk factors or had multiple neurological deficits. This explains why the children in this category correlated highly with abnormal EEG. This suggests that CWE with multiple etiological factors should also be prioritized in EEG appointments in resource-poor countries.
Age has been reported in several studies ${ }^{8-11}$ as a clinical factor associated with inter-ictal epileptiform abnormality and paediatric age group is a predictor of EEG abnormality. This study detected a higher proportion of EEG abnormalities in the age group $<5$ years but the lack of association between age groups and abnormal EEG is consistent with the report by Aina et al. ${ }^{12}$ but differs from earlier studies $^{8-11}$ although those studies were not specifically done in children alone. In a multicentre study ${ }^{13}$ with a large sample size and sufficient power to measure associations between clinical categories, age was not one of the factors associated with abnormal or epileptiform EEG. More so, the lack of association between abnormal EEG and age at the time of EEG found in this present study is consistent with some prior reports ${ }^{12,13}$ in the literature. However, the study by Drury et al. ${ }^{14}$ was done in patients older than 65 years and the study by Selvitelli et al. ${ }^{15}$ has a mean age of 40.33 years and range of 16-80 years. Therefore, it is possible that across all age groups, the paediatric age group is a predictor of EEG abnormality but within the paediatric age group, age is not associated with abnormal EEG.

The cohort in this present study had more males than females and explains why a greater number of males with abnormal EEG were found but the proportion with abnormal EEG is similar to that of females. The lack of association between abnormal EEG and gender in this study is consistent with other reports..$^{8-10,15}$ However, the study by Owolabi et al. ${ }^{11}$ though not done exclusively in children reported female gender as an independent predictor of interictal EEG abnormality. The reasons for these conflicting findings are yet to be elucidated.

Among other factors, the recording of interictal epileptiform activity is dependent on seizure type and specific epileptiform patterns on interictal EEG are most commonly absent in patients with focal epilepsy of extra-temporal origin. ${ }^{16}$ Additionally, an EEG is more likely to show epileptiform abnormalities in untreated epileptic spasms, Idiopathic Generalized Epilepsies (IGEs) especially absence seizures and convulsive and non-convulsive status epilepticus while a single EEG is more likely to be normal (80\%) than abnormal in mesial frontal, mesial temporal and mesial occipital lobe epilepsies (focal epilepsies). ${ }^{4}$ It was surprising that there was no proven relationship between the occurrence of EEG abnormalities and clinical seizure types in this study and this differs from earlier reports from Nigeria by Falope et al. ${ }^{9}$ though consistent with the findings by Monif and Seneviratne. ${ }^{8}$

Various antiepileptic drugs have variable suppressive effects on epileptiform activity on EEG and patients who have not commenced any AED therapy may be more likely to have epileptiform or abnormal EEG. ${ }^{17}$ In this study, there was a small difference (less than $10 \%$ ) in the proportions of patients with or without AEDs who had abnormal EEG and it was not surprising that this difference did not reach statistical significance. This could be attributed to the small sample size of this study and the low sensitivity of an initial EEG. The lack of association between the occurrence of abnormal EEG and AED therapy reported in this study agrees with an earlier study in Nigeria by Falope et al. ${ }^{9}$ However, the study by Monif and Seneviratne ${ }^{8}$ with a large statistical power found no AED therapy as one of the factors significantly associated with epileptiform EEG and posited that patients who are not on AEDs are more likely to have epileptiform EEG.

Sleep remains the most effective EEG activation procedure that produces the highest yield of epileptiform abnormalities especially in younger population. ${ }^{18}$ Thus, it was surprising that the association between the occurrence of abnormal EEG and sleep was not statistically significant. However findings cannot be generalized based on drug-induced sleep in the present study. Most of 
the earlier reports $8,9,11$ did not evaluate this factor in relation to the occurrence of abnormal EEG and so further studies are needed to verify this finding.

\section{Limitations}

Due to lack of information from records (retrospective design of the study), this study did not investigate the relationship between occurrence of abnormal EEG and frequency of seizures, duration of epilepsy and proximity of EEG to last seizure episode - clinical factors earlier reported to be associated with the occurrence of abnormal or epileptiform EEG. This study evaluated initial (single) routine EEGs but serial EEGs would have augmented the yield and strengthened associations between the variables. Furthermore, the relatively small sample of this study does not provide sufficient statistical power to measure associations between clinical variables.

\section{Conclusions}

The clinical neurological state and the aetiology of epilepsy in children were positively associated with the occurrence of abnormalities and yield of interictal scalp EEG. Children with epilepsy who are neurologically abnormal or have multiple aetiological factors could be prioritized in EEG appointments in resource-poor settings. Further research in children with epilepsy is needed to confirm these findings.

\section{References}

1. Pandey S. Challenges in Neurological Practice in Developing Countries. Indian J Public Health 2012;56:227- 230

2. Camfield P, Camfield C. Incidence, prevalence and aetiology of seizures and epilepsy in children. Epileptic disorders 2015; 17:117-23.

3. Ughasoro MD, Onwujekwe OE, Ojinnaka NC. Economic cost of treatment of childhood epilepsy in Enugu, Southeast Nigeria. Intl J Technol Assessment Health Care 2014;30:1-6.

4. Panayiotopoulos CP. EEG and brain imaging. In: Panayiotopoulos CP (eds). A clinical guide to epileptic syndromes and their treatment. 2nd ed. London: Springer Healthcare Ltd; 2010. pp. 147-71.

5. Mclane HC, Berkowitz AL, Patenaude BN et al. Availability, accessibility, and affordability of neurodiagnostic tests in 37 countries. Neurology® 2015;85:1614-22.
6. Fisher RS, Cross JH, D’Souza C, et al. Instructional Manual for the ILAE 2017 operational classification of seizure types. Epilepsia 2017;58:531-42

7. Falco-Walter JJ, Scheffer IE, Fisher RS. The new definition and classification of seizures and epilepsy. Epilepsy Res 2018;139:73-9.

8. Monif M, Seneviratne U. Clinical factors associated with the yield of routine outpatient scalp electroencephalograms: A retrospective analysis from a tertiary hospital. J Clin Neurosci 2017;45:110-4

9. Falope ZF, Ogunniyi A, Osuntokun BO. Factors associated with epileptiform EEG patterns in Nigerian epileptics. East Afr Med J 1993;70:294-6

10. Baldin E, Hauser WA, Buchhalter JR, Hesdorffer DC, Ottman R. Yeild of epileptiform EEG abnormalities in incident unprovoked seizures: a population-based study. Epilepsia 2014;55:1389-98.

11. Owolabi LF, Sale S, Owolabi SD, Nalado A, Umar M, Taura AA. Electroencephalography abnormalities in generalized epilepsy and their predictors: A multi-centre experience. Ann Afr Med 2018;17:64-9.

12. Aina OF, Ogun OC, Ladapo HTO, et al. Clinical neuropsychiatric correlates and EEG findings among children with developmental disorders in Lagos, Nigeria. Afr J Psychiatry 2008;11:123-7.

13. Kariuki SM, White S, Chengo E, et al. Electroencephalographic features of convulsive epilepsy in Africa: A multicentre study of prevalence, pattern and associated factors. Clinical Neurophysiol 2016;127:1099-1107.

14. Drury I, Beydoun A. Interictal epileptiform activity in elderly patients with epilepsy. Electroencephalogr Clin Neurophysiol 1998;106:369-73.

15. Selvitelli MF, Walker LM, Schomer DL, Chang BS. The relationship of interictal epileptiform discharges to clinical epilepsy severity: A study of routine EEGs and review of the literature. J Clin Neurophysiol 2010;27:87-92.

16. Cascino GD, Herkes GK. Interpretation of Interictal EEG. In: Elaine Wyllie (eds). The treatment of Epilepsy: Principles and practice. Philadelphia, Lea \& Febiger; 1993: p.249-61.

17. Schmidt D. The influence of antiepileptic drugs on the electroencephalogram: a review of controlled clinical studies. Electroencephalogr Clin Neurophysiol Suppl 1982;36:453-66.

18. Baldin E, Hauser WA, Buchhalter JR, et al. Utility of EEG activation procedures in Epilepsy: A population-based study. J Clin Neurophysiol 2017;34:512-9 